\title{
Alternating Error Effects on Decomposition Method in Function Generation Synthesis
}

\author{
O.W. Maaroof ${ }^{1}$, M.İ.C Dede ${ }^{1}$ and G. Kiper ${ }^{1}$ \\ IIzmir Institute of Technology, Turkey,e-mails: omarmaaroof@iyte.edu.tr, \\ candede@iyte.edu.tr,gokhankiper@iyte.edu.tr
}

\begin{abstract}
In approximate function generation synthesis methods, error between the desired function's output and designed mechanism's output oscillate about zero error while crossing the zero error margin at precision points. The common goal of these methods is to minimize the error within the selected working region of the mechanism. For mechanisms like Bennett overconstrained six-revolute jointed linkages that have relatively large number of construction parameters, it is a difficult task to solve for them at once. Decomposition method enables to divide such linkages into two loops and independently solve for each loop with less construction parameters. Although some approximation methods are proven to produce smaller errors than others for a single-loop synthesis, in this work, it is shown that smaller errors are not guaranteed for a certain method when used along with decomposition method. Numerical examples indicate that in decomposition method, more attention should be given to the alternation of the error of each decomposed mechanism, rather than the approximation method used.
\end{abstract}

Key words: Bennett double spherical linkages, input-output equation, decomposition method, mechanism synthesis, overconstrained mechanism.

\section{Introduction}

The results of Sarrus [1] on planar-planar six-bar linkage was studied further and generalized into double-spherical six-bar and plano-spherical six-bar linkages by Bennett [2]. Alternative ways to develop these overconstrained linkages were studied such as; linkages obtained through intersecting Euclidean subspaces [3], or using double-Hooke's joint linkages to develop double-spherical six-bar linkage [4].

Several methods were proposed to design spherical four-bar mechanisms through kinematic synthesis procedures for function generation $[5,6,7]$. Best design is considered to be the one that results in smaller amount of errors for the desired function. The work presented in this paper is on utilizing the decomposition method for the function generation synthesis of Bennett six-revolute jointed (6R) overconstrained linkages to decrease the error range of the desired function. 
In a previous work of the authors on kinematic synthesis of double spherical sixbar mechanisms, extensive description of the mechanism was given [8]. The decomposition method was applied to decompose the function into two parts and therefore synthesize each spherical four-bar loops by introducing a passive joint as illustrated in Figure 1. The input-output (I/O) equation was derived for four-construction-parameter synthesis for both parts of the linkage and only interpolation approximation method (IPM) was used. This idea was extended in $[9,10]$ for all types of Bennett overconstrained 6R linkages and I/O equations were derived for function generation using Chebyshev approximation. The work presented in this paper is a continuation of efforts for understanding the inconsistency in decreasing errors by using various methods for the double-spherical six-bar linkage.

A single four-bar linkage designed with least-squares approximation (LSA) is expected to result smaller error range in function generation with respect to the results with IPM given that a wise selection of number of design points was made. However, in this paper by testing the methods with different functions to be generated, it is shown that when using the decomposition method, it is not the synthesis method that has domination on the total error range but direction of the error alternation about the zero-error line. Therefore, in decomposition method, special care should be given to the alternation of error range rather than the approximate synthesis method.

In this paper, double-spherical $6 \mathrm{R}$ linkage is selected to evaluate the condition for minimizing the maximum errors in the decomposition method. However, this is also applicable to the function generation synthesis of the plano-spherical or doubleplanar 6R linkages as well. Derivation of the I/O equations and description of the double-spherical 6R linkage for four angular construction parameters per loop are given in full detail in [8]. Also, IPM is described in [8]. LSA method used in this work is an application of the method described in [7]. In this paper, a new error calculation technique is introduced and the procedures are described along with numerical example calculations. Finally, numerical example results are discussed and future works are addressed.

\section{Derivation of the $\mathrm{I} / \mathrm{O}$ equations for the double- spherical 6R linkage}

The I/O relationship of the double-spherical mechanism can decomposed into two parts. As shown in Figure 1, input angle $\phi$ can be considered as input of the first spherical four-bar loop and also the input of the double spherical linkage, while its output angle $\widetilde{\psi}$ is associated with the passive joint. At the same time, the passive joint variable $\widetilde{\psi}$ is the input of the second spherical four-bar mechanism. Finally, $\psi$, is the output of both second spherical four-bar loop and output of the double-spherical six-bar linkage. 
Synthesis procedure of spherical four-bar mechanisms are well studied and as a result, I/O equations for providing geometric constraints between links (twist angle between joints) can be derived and simplified by using an algebraic method. Transformation of unit vector equation as described in [11] is used to find the relationship between the unit vectors $\left(\mathbf{S}_{\mathrm{i}}, \mathbf{S}_{\mathrm{j}}\right.$, and $\left.\mathbf{S}_{\mathrm{k}}\right)$ shown in Figure 1.

In order to clarify the generation of the $\mathrm{I} / \mathrm{O}$ equations, following equalities are provided: $\mathbf{S}_{\mathrm{i}}\left(\mathrm{l}_{\mathrm{i}}, \mathrm{m}_{\mathrm{i}}, \mathrm{n}_{\mathrm{i}}\right)$ that rotates around $\mathbf{S}_{\mathrm{j}}\left(\mathrm{l}_{\mathrm{j}}, \mathrm{m}_{\mathrm{j}}, \mathrm{n}_{\mathrm{j}}\right)$ by angle $\alpha_{\mathrm{i}, \mathrm{k}}, \mathbf{S}_{\mathrm{k}}\left(\mathrm{l}_{\mathrm{k}}, \mathrm{m}_{\mathrm{k}}, \mathrm{n}_{\mathrm{k}}\right)$ can be calculated as $\mathbf{S}_{\mathrm{k}}=\mathbf{S}_{\mathrm{i}} \cos \alpha_{\mathrm{i}, \mathrm{k}}+\mathbf{S}_{\mathrm{ji}} \sin \alpha_{\mathrm{i}, \mathrm{k}}$ where, $\mathbf{S}_{\mathrm{ji}}=\mathbf{S}_{\mathrm{j}} \times \mathbf{S}_{\mathrm{i}}$. Revolute joint axes of the mechanism are described via joint unit vectors (Fig. 1). To find all vectors is significant for formulating the I/O equations. First, $\mathbf{S}_{1}=(1,0,0)$ and $\mathbf{S}_{2}=$ $(0,0,1)$ are selected as unit vectors of an orthogonal coordinate frame. The other vectors are found accordingly making use of calculation procedure given above.

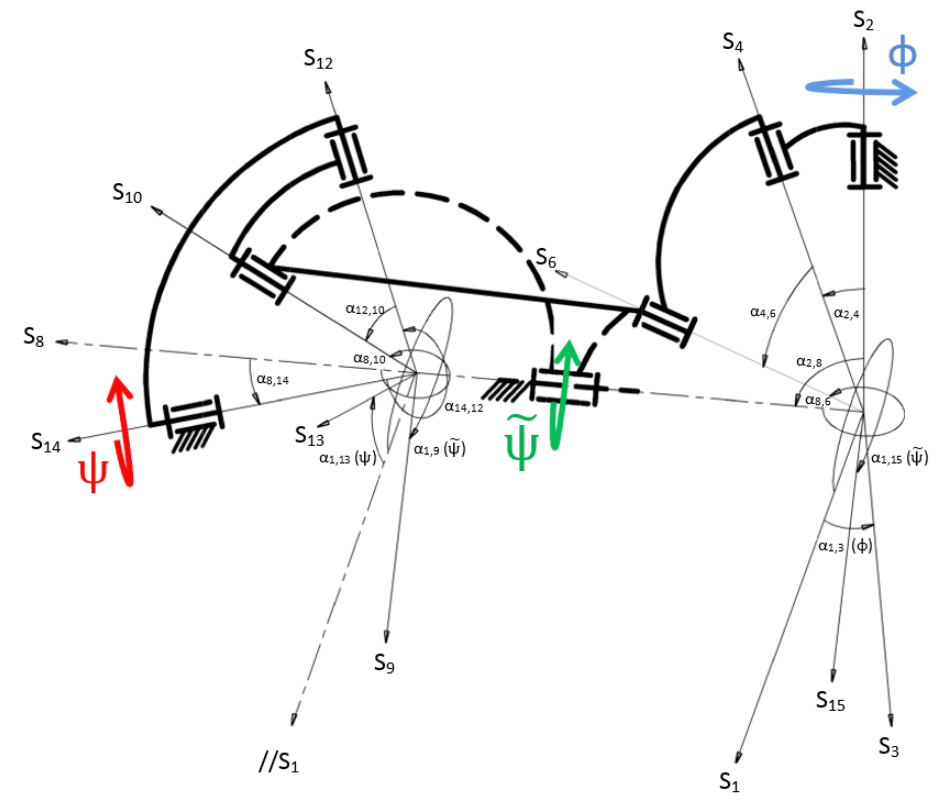

Fig. 1 Double-spherical six-bar linkage mechanism with joint axes.

The I/O equation of the first four-bar is calculated as $(\mathrm{C} \alpha=\cos \alpha$ and $\mathrm{S} \alpha=\sin \alpha)$ : $\mathbf{S}_{6} \cdot \mathbf{S}_{4}=C \alpha_{4,6}$. Substituting values of $\mathbf{S}_{4}$ and $\mathbf{S}_{6}$ and manipulating results in:

$-\mathrm{C} \alpha_{4,6}+\mathrm{C} \alpha_{2,4} \mathrm{C} \alpha_{2,8} \mathrm{C} \alpha_{8,6}+\mathrm{C} \alpha_{1,3} \mathrm{C} \alpha_{8,6} \mathrm{~S} \alpha_{2,4} \mathrm{~S} \alpha_{2,8}+\mathrm{C} \alpha_{1,3} \mathrm{C} \alpha_{1,15} \mathrm{C} \alpha_{2,8} \mathrm{~S} \alpha_{2,4} \mathrm{~S} \alpha_{8,6}-$
$\mathrm{C} \alpha_{1,15} \mathrm{C} \alpha_{2,4} \mathrm{~S} \alpha_{2,8} \mathrm{~S} \alpha_{8,6}+\mathrm{S} \alpha_{1,3} \mathrm{~S} \alpha_{1,15} \mathrm{~S} \alpha_{2,4} \mathrm{~S} \alpha_{8,6}=0$

where the input $\phi$ is $\alpha_{1,3}$ and output $\widetilde{\psi}$ is $\alpha_{1,15}$. The I/O equation for the second fourbar is derived from $\mathbf{S}_{\mathbf{1 2}} \cdot \mathbf{S}_{\mathbf{1 0}}=\mathrm{C} \alpha_{10,12}$. Substituting values of $\mathbf{S}_{12}$ and $\mathbf{S}_{10}$ and manipulating results in: 


$$
\begin{gathered}
-\mathrm{C} \alpha_{10,12}+\mathrm{C} \alpha_{8,10} \mathrm{C} \alpha_{8,14} \mathrm{C} \alpha_{14,12}+\mathrm{C} \alpha_{14,12} \mathrm{C} \alpha_{1,9} \mathrm{~S} \alpha_{8,14} \mathrm{~S} \alpha_{8,10}+ \\
\mathrm{C} \alpha_{8,14} \mathrm{C} \alpha_{1,9} \mathrm{C} \alpha_{1,13} \mathrm{~S} \alpha_{8,10} \mathrm{~S} \alpha_{14,12}-\mathrm{C} \alpha_{8,10} \mathrm{C} \alpha_{1,13} \mathrm{~S} \alpha_{8,14} \mathrm{~S} \alpha_{14,12} \\
+\mathrm{S} \alpha_{1,15} \mathrm{~S} \alpha_{1,13} \mathrm{~S} \alpha_{8,10} \mathrm{~S} \alpha_{14,12}=0
\end{gathered}
$$

where the input $\widetilde{\psi}$ is $\alpha_{1,9}$, which is also the output for the first loop, $\alpha_{1,15}$. The output for the second loop, $\psi$, is $\alpha_{1,13}$, which is also the output of the double-spherical $6 \mathrm{R}$ linkage.

\section{A new way of error calculation}

A function $\mathrm{y}=\hat{\mathrm{f}}(\mathrm{x})$ to be generated can be decomposed into two as $\tilde{\mathrm{y}}=\hat{\mathrm{h}}(\mathrm{x}), \mathrm{y}=$ $\hat{\mathrm{g}}(\tilde{\mathrm{y}})$ and $\mathrm{y}=\hat{\mathrm{g}}(\hat{\mathrm{h}}(\mathrm{x}))=\hat{\mathrm{f}}(\mathrm{x})$ where $\mathrm{x}$ and $\mathrm{y}$ are the input and output of the function, where as $\tilde{y}$ is and intermediate variable. For approximating these functions, the $\mathrm{I} / \mathrm{O}$ equation of the double-spherical linkage can be written in polynomial form in terms of the construction parameters. Then the I/O equations can be determined for the first and second spherical four-bar loops and the double-spherical linkage as: $\widetilde{\psi}=\mathrm{h}(\phi, \overline{\mathrm{c}}), \psi=\mathrm{g}(\widetilde{\psi}, \overline{\mathrm{d}})$ and $\psi=\mathrm{g}(\mathrm{h}(\phi, \overline{\mathrm{c}}), \overline{\mathrm{d}})=\mathrm{f}(\phi, \overline{\mathrm{c}}, \overline{\mathrm{d}})$. These functions represent the generated output angles for the designed loops, where $\overline{\mathrm{c}}=$ $\left\{\alpha_{2,8}, \alpha_{2,4}, \alpha_{8,6}, \alpha_{4,6}\right\}$ and $\bar{d}=\left\{\alpha_{8,14}, \alpha_{14,12}, \alpha_{8,10}, \alpha_{12,10}\right\}$ are vectors containing the construction parameters of the first and second four-bar loops, respectively. The input/outputs of $\mathrm{f}, \mathrm{g}$, $\mathrm{h}$ functions are linearly related with the input/outputs of the $\hat{\mathrm{f}}, \hat{\mathrm{g}}, \hat{\mathrm{h}}$ functions as $\phi=a_{1} \mathrm{x}+\mathrm{a}_{2}, \widetilde{\psi}=\mathfrak{b}_{1} \tilde{\mathrm{y}}+\hat{b}_{2}$ and $\psi=\mathrm{b}_{1} \mathrm{y}+\mathrm{b}_{2}$, where the coefficients $a_{1}, a_{2}, \mathfrak{b}_{1}, \mathfrak{b}_{2}, \mathrm{~b}_{1}, \mathrm{~b}_{2}$ depend on the limits of $\mathrm{x}, \tilde{\mathrm{y}}, \mathrm{y}, \phi, \widetilde{\psi}$ and $\psi$. The cumulative errors in the output angles of the two loops were defined in terms of the input angles in [8] as follows:

$$
\begin{gathered}
\grave{\mathrm{e}}_{1}=\sum_{\phi=72}^{180}\left|\mathrm{~h}(\phi, \overline{\mathrm{c}})-\left(\mathrm{b}_{1} \hat{\mathrm{h}}\left(\frac{\phi-\mathrm{a}_{2}}{\grave{\mathrm{a}}_{1}}\right)+\mathrm{b}_{2}\right)\right| \\
\grave{\mathrm{e}}_{2}=\sum_{\widetilde{\psi}=18}^{108}\left|\mathrm{~g}(\widetilde{\Psi}, \overline{\mathrm{d}})-\left(\mathrm{b}_{1} \hat{\mathrm{g}}\left(\frac{\widetilde{\Psi}-\dot{\mathrm{b}}_{2}}{\grave{\mathrm{b}}_{1}}\right)+\mathrm{b}_{2}\right)\right|
\end{gathered}
$$

where the angle limits $72^{\circ} \leq \phi \leq 180^{\circ}$ and $18^{\circ} \leq \widetilde{\psi} \leq 108^{\circ}$ are sample values and can be selected arbitrarily by the designer considering the design constraints, if there is any. In Eq. (5), $e_{t}$ is the total error for the double-spherical mechanism. One can also use the root mean square (RMS) of the errors as well.

$$
\mathrm{e}_{\mathrm{t}}=\sum_{\phi=72}^{180}\left|\mathrm{~g}(\mathrm{~h}(\phi, \overline{\mathrm{c}}), \overline{\mathrm{d}})-\left(\mathrm{b}_{1} \hat{\mathrm{g}}\left(\widehat{\mathrm{h}}\left(\frac{\phi-\dot{a}_{2}}{\mathrm{a}_{1}}\right)\right)+\mathrm{b}_{2}\right)\right|
$$


The total error $e_{t}$ is composed of both first and second part loop errors. Each error has a real value for a specific double-spherical linkage's input value of $\phi$ angle. The error values inside the absolute sign in Eqs. (3), (4) and (5) can be positive or negative and the combined error of the two loops can have a very low value when corresponding individual errors in the loops have opposite signs. To calculate the mean of the total error, $\mathrm{e}_{\mathrm{tm}}$ in terms of the loop errors, $\grave{\mathrm{e}}_{2}$ can be modified to be a function of $\phi$ angle instead of $\widetilde{\psi}$ angle as shown below. To accommodate this procedure, the errors due to the first loop are modified as:

$$
\begin{gathered}
\mathrm{e}_{1}(\phi)=\mathrm{b}_{1}\left(\hat{\mathrm{g}}\left(\frac{\mathrm{h}(\phi, \bar{c})-\mathfrak{b}_{2}}{\mathfrak{b}_{1}}\right)-\hat{\mathrm{f}}\left(\frac{\phi-\mathfrak{a}_{2}}{\dot{a}_{1}}\right)\right) \\
\mathrm{e}_{1 \mathrm{~m}}=\sum_{\phi=72}^{180}\left|\mathrm{e}_{1}(\phi)\right| / \mathrm{h}
\end{gathered}
$$

where $\mathrm{e}_{1 \mathrm{~m}}$ is the mean error in $\psi$ due the first spherical loop expressed in terms of angle $\phi$ with a sampling rate $\mathrm{s}=1^{\circ}$, i.e. $\phi=72^{\circ}, 73^{\circ}, \ldots, 180^{\circ}$. One can use finer or coarse sampling rate. $n=(180-72+1) / s=109$ for $s=1$. In defining $e_{1}(\phi)$ the second spherical four-bar loop is assumed to be a perfect mechanism resulting no error in $\psi$. For error due to the second loop

$$
\begin{gathered}
\mathrm{e}_{2}(\phi)=g\left(\left(\hat{\mathrm{b}}_{1} \hat{\mathrm{h}}\left(\frac{\phi-\dot{a}_{2}}{\dot{a}_{1}}\right)+\hat{b}_{2}\right), \overline{\mathrm{d}}\right)-\left(\mathrm{b}_{1} \hat{\mathrm{g}}\left(\hat{\mathrm{h}}\left(\frac{\phi-\dot{a}_{2}}{\dot{a}_{1}}\right)\right)+\mathrm{b}_{2}\right) \\
\mathrm{e}_{2 \mathrm{~m}}=\sum_{\phi=72}^{180}\left|\mathrm{e}_{2}(\phi)\right| / \mathrm{h}
\end{gathered}
$$

where $\mathrm{e}_{2 \mathrm{~m}}$ is the mean error in $\psi$ due the second spherical loop, where the first spherical four-bar loop is assumed to be a perfect mechanism with resulting no errors in $\widetilde{\psi}$. Finally for the double-spherical linkage

$$
\mathrm{e}_{\mathrm{tm}}=\sum_{\phi=72}^{180}\left|\mathrm{e}_{1}^{\prime}+\mathrm{e}_{2}^{\prime}\right| / \mathrm{n}
$$

where $e_{t m}$ is the total mean error in $\psi$. The total error calculated in Eq. (10) is the same total error calculated in Eq. (5). However, new calculation method of the two error values for two decomposed loops will enable to visualize how the errors are combined to result in the total error.

\section{Numerical examples and results}

In this section, an exponential and a power function are selected for the function to be generated so that they resemble a nonlinear relation between the input and the output. For function generation of the both functions, IPM and LSA are used. 
For the numerical examples, the first function to be generated by the doublespherical 6R linkage is $y=x^{1.3}$. This function is decomposed into two as $y^{\prime}=x^{0.8}$ and $y=y^{1.625}$. One can also choose some other type of decomposition between the two functions (such as $y=x^{2.6}$ and $y=y^{0.5}$ ). Second function to be generated is $\mathrm{y}=\mathrm{e}^{2 \mathrm{x}}$, which is decomposed into two as $y=\mathrm{e}^{1.2 \mathrm{x}}$ and $\mathrm{y}=\dot{y}^{(2 / 1.2)}$. Once again, other options for decomposing the function are possible. For both of the functions, input angle limits are selected as $72^{\circ} \leq \phi \leq 180^{\circ}$. Intermediate angle limits are selected as $18^{\circ} \leq \widetilde{\psi} \leq 108^{\circ}$. Output angle limits are selected as $72^{\circ} \leq \psi \leq 180^{\circ}$. These limits can be changed to decrease the error or comply with some design constraints.

In IPM, precision points are distributed in the range of input angles for each mechanism using equal spacing method in range of $\mathrm{x}$ from $\mathrm{x}_{0}=1$ to $\mathrm{x}_{\mathrm{m}}=2$ for the first loop and from $y_{0}=1^{0.8}$ to $y_{m}=2^{0.8}$ for the second loop in numerical example one and $y_{0}=\mathrm{e}^{1.2}$ to $\hat{y}_{\mathrm{m}}=\mathrm{e}^{2.4}$ for the second loop in numerical example two, and $\mathrm{x}_{\mathrm{n}}=\mathrm{x}_{\mathrm{n}-1}+\delta$ for $\mathrm{n}=1,2,3$ where $\delta=\left(\mathrm{x}_{\mathrm{m}}-\mathrm{x}_{0}\right) / 3$. Output values at the precision points can be calculated as $y_{i}=\hat{f}\left(x_{i}\right)$ for $i=0,1,2,3$.

To find the LSA solution for the first loop, the summation is done for $72^{\circ} \leq \phi \leq$ $180^{\circ}$ by s $=1^{\circ}$ step $\rightarrow \mathrm{n}=109$ ( $\mathrm{n}$ is the number of design points). To find the LSA solution for the second part, the previous procedure is repeated with $\widetilde{\psi}$ ranging from $18^{\circ}$ to $108^{\circ}$ by $1^{\circ}$ step $\rightarrow \mathrm{n}=91$ in numerical example one and by $7^{\circ}$ step $\rightarrow \mathrm{n}=$ 13 in numerical example two. The step size in numerical example two is increased to $7^{\circ}$ in order to get a real solution.

Tables 1 and 2 present mean errors for the first four-bar spherical loop and the second four-bar spherical loop and mean total error of the double-spherical linkage obtained using both IPM and LSA for generating two different functions. The designed construction parameters of the mechanisms are also presented in the tables, where the superscripts indicate the first or second loop of the decomposed linkage.

Table 1. The calculated mean errors and construction parameters for $y=x^{1.3}$

\begin{tabular}{ccccccccc}
\hline & \multicolumn{7}{c}{ Mean error values } & \multicolumn{7}{c}{ Construction Parameters (rad) } \\
\hline & $I P M$ & $L S A$ & & $I P M^{l}$ & $L S A^{l}$ & & \multicolumn{1}{c}{$I P M^{2}$} & $L S A^{2}$ \\
\hline $\mathbf{e}_{\mathbf{1 m}}$ & $7.6 \mathrm{e}^{-4}$ & $5.5 \mathrm{e}^{-4}$ & $\boldsymbol{\alpha}_{\mathbf{2 , 8}}$ & 0.422 & 0.430 & $\boldsymbol{\alpha}_{\mathbf{8 , 1 4}}$ & 0.825 & 0.729 \\
$\mathbf{e}_{\mathbf{2 m}}$ & 0.0019 & 0.0013 & $\boldsymbol{\alpha}_{\mathbf{2 , 4}}$ & 0.803 & 0.823 & $\boldsymbol{\alpha}_{\mathbf{1 4 , 1 2}}$ & -0.720 & -0.800 \\
$\mathbf{e}_{\mathbf{t m}}$ & 0.0017 & 0.0015 & $\boldsymbol{\alpha}_{\mathbf{8 , 6}}$ & 1.177 & 1.203 & $\boldsymbol{\alpha}_{\mathbf{8 , 1 0}}$ & 0.504 & 0.543 \\
& & & $\boldsymbol{\alpha}_{\mathbf{4 , 6}}$ & 1.164 & 1.175 & $\boldsymbol{\alpha}_{\mathbf{1 2 , 1 0}}$ & 0.921 & 0.979 \\
\hline
\end{tabular}

Table 2. The calculated mean errors and construction parameters for $y=e^{2 x}$

\begin{tabular}{|c|c|c|c|c|c|c|c|c|}
\hline & \multicolumn{2}{|c|}{ Mean error values } & \multicolumn{6}{|c|}{ Construction Parameters (rad) } \\
\hline & $I P M$ & $L S A$ & & $I P M^{l}$ & $L S A^{1}$ & & $I P M^{2}$ & $L S A^{2}$ \\
\hline $\mathbf{e}_{1 \mathrm{~m}}$ & 0.0020 & 0.0015 & $\alpha_{2,8}$ & 0.376 & 0.346 & $\alpha_{8,14}$ & 1.597 & 0.992 \\
\hline $\mathbf{e}_{2 \mathrm{~m}}$ & 0.0033 & 0.0024 & $\alpha_{2,4}$ & -1.125 & -1.028 & $\alpha_{14,12}$ & -0.255 & -0.405 \\
\hline \multirow[t]{2}{*}{$\mathbf{e}_{\mathrm{tm}}$} & 0.0019 & 0.0024 & $\alpha_{8,6}$ & -0.771 & -0.693 & $\alpha_{8,10}$ & 0.195 & 0.296 \\
\hline & & & $\alpha_{4,6}$ & 0.842 & 0.767 & $\alpha_{12,10}$ & 1.430 & 0.853 \\
\hline
\end{tabular}


Each loop's error throughout the input angle range (about 1.26 to $3.14 \mathrm{rad}$ ) is given in Figs. 2 and 3 for the generation of $y=x^{1.3}$ and $y=e^{2 x}$, respectively.

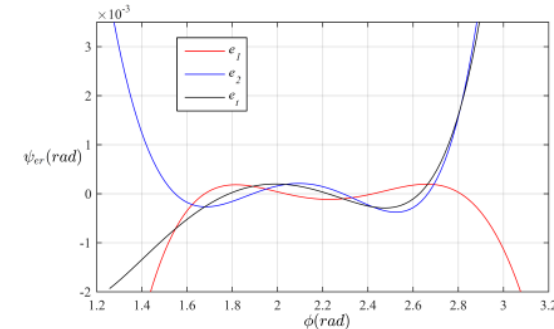

(a)

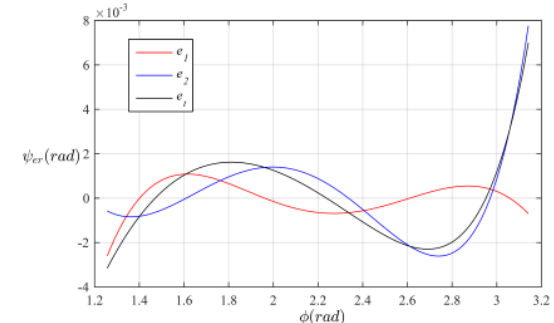

(b)

Fig. 2 Error of output angle for $y=x^{1.3}$ by using (a) IPM (b) LSA.

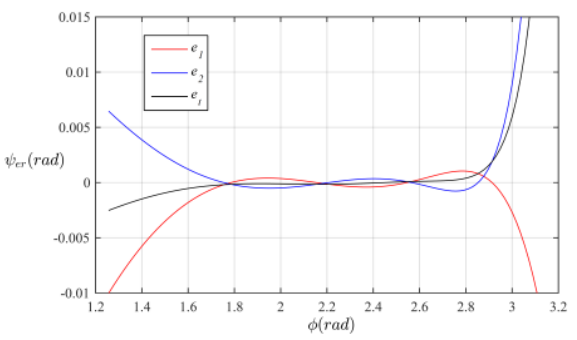

(a)

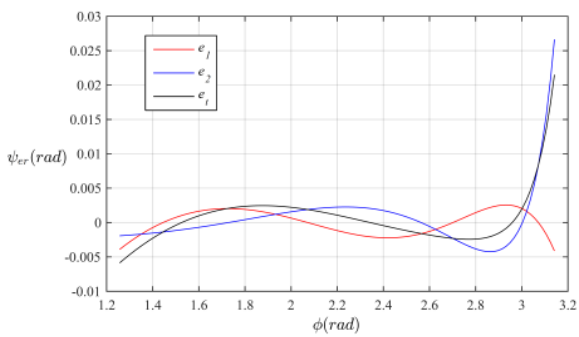

(b)

Fig. 3 Error of output angle for $y=e^{2 x}$ by using (a) IPM (b) LSA.

\section{Conclusions}

It is observed in both case studies with different functions that, in the independent kinematic synthesis of the first and the second spherical four-bar loops, smaller error ranges were obtained with LSA with respect to IPM. Nevertheless, total error calculated with the LSA for the double-spherical 6R linkage is smaller in the first example and larger in the second example with respect to the results obtained with IPM.

In order to serve for better understanding of the inconsistency, a new error calculation procedure is defined during the design of a single spherical four-bar mechanism. Resultant error variations are analyzed in a single plot to indicate the error oscillation. Also, it is shown that the total error of the double-spherical 6R mechanism can be calculated by adding up the total errors calculated after the first fourbar loop is designed and the total errors calculated after the second four-bar loop is designed.

Evaluating the numerical results, it is concluded that the alternating total errors for the synthesis of the first and second spherical four-bar loops can result in smaller 
total error for the kinematic synthesis of the double-spherical six-bar linkage regardless of the approximation method used. In order to result in a minimized error range in the obtained function, independent of the method of approximate synthesis chosen, the error alternation plots for both decomposed mechanisms should be inspected to ensure an opposite direction of alternation of errors. Investigating a way to guarantee error alternation is the scope of a future work on this area.

Although, synthesis procedure presented in this paper can be carried out for more examples with different ranges, the numerical examples presented in this paper provide basis for the above conclusions.

Acknowledgments The authors would like to thank to The Scientific and Technological Research Council of Turkey for funding the research presented in this work (grant number 115E726). Also, the authors are grateful for the guidance of Prof. Rasim Alizade.

\section{References}

1. Sarrus, P.T.: Note sur la Transformation des Mouvements Rectilignes Alternatifs, en Mouvements Circulaires; et Reciproquement. Acad. Sci. 36, 1036-1038 (1853)

2. Bennett, G.T.: The Parallel Motion of Sarrus and Some Allied Mechanisms. Philosophy Mag. 6th series, 9, 803-810 (1905)

3. Baker, J.E.: Displacement-Closure Equations of the Unspecialized Double Hooke's-Joint Linkage, Mech. Mach. Theory, 3, 1127-1144 (2002)

4. Alizade, R.I., Aydazade, K.P. and Novruzbekov, I.G.: Analysis and Synthesis of Planar Mechanisms by Using Decomposition Method. J. Mechanics of Mach. The Academy of Sciences of the USSR, 57, 26-32 (1980)

5. Murray, A.P. and McCarthy, J.M.: A Linkage Map for Spherical Four Position Synthesis. In: Proceedings of ASME Tech. Conf., Boston, MA, pp. 833-844 (1995)

6. Cervantes-Sánchez, J.J., Medellín-Castillo, H.I., Rico-Martínez, J.M. and González-Galván, E.J.: Some Improvements on the Exact Kinematic Synthesis of Spherical 4R Function Generators. Mech. Mach. Theory, 44, 103-121 (2009)

7. Alizade, R.I. and Gezgin, E.: Synthesis of Function Generating Spherical Four Bar Mechanism for the Six Independent Parameters. Mech. Mach. Theory, 46, 1316-1326 (2011)

8. Maaroof, O.W. and Dede, M.I.C.: Kinematic Synthesis of Over-Constrained Double-Spherical Six-Bar Mechanism. Mech. Mach. Theory, 73, 154-168 (2014)

9. Alizade, R.I., Kiper, G., Dede, M.I.C. and Uzunoglu, E.: Derivation of Input/Output Relationships for the Bennett 6R Linkages Based on the Method of Decomposition. In: V. Petuya et al. (Eds.), New Adv. in Mech., Transmissions and Appl. Springer, pp. 225-231 (2014)

10. Alizade, R.I., Kiper, G., Dede, M.I.C. and Bağdadioğlu, B.: Function synthesis of Bennett 6R mechanisms using Chebyshev approximation. Mech. Mach. Theory, 81, 62-78 (2014)

11. Alizade, R.I.: Structural Synthesis of Robot Manipulators. In: Proceedings of Int. Symp. Mech. Mach. Sci., Izmir, Turkey, pp. 11-32 (2010) 